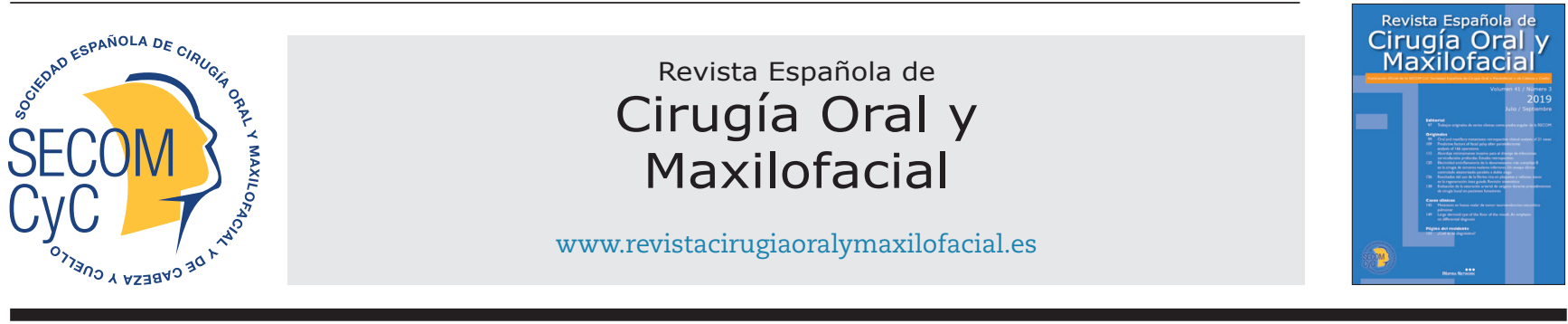

\title{
Original
}

\section{Efectividad antinflamatoria de la dexametasona más complejo B en la cirugía de terceros molares inferiores. Un ensayo clínico controlado aleatorizado paralelo a doble ciego}

\author{
Marco Reátegui-Navarro ${ }^{a}$, Gissela Gálvez-Cubas ${ }^{b}$ y Heber Arbildo-Vega ${ }^{c}$
}

${ }^{a}$ Facultad de Estomatología, Universidad Nacional de Trujillo, Trujillo, Perú. ${ }^{b}$ Escuela de Estomatología, Universidad Privada Antenor Orrego, Trujillo, Perú. 'Escuela de Estomatología, Universidad Señor de Sipán, Chiclayo, Perú; Escuela de Odontología, Universidad Particular de Chiclayo, Chiclayo, Perú; Centro de Salud Odontológico San Mateo, Trujillo, Perú.

\section{INFORMACIÓN DEL ARTÍCULO}

Historia del artículo:

Recibido el 14 de septiembre de 2017 Aceptado el 13 de abril de 2018

\section{Palabras clave:}

Dexametasona, tercer molar, cirugía oral, edema, efecto antinflamatorio, ensayo clínico.

\section{R E S U M E N}

\begin{abstract}
Objetivo: Determinar la efectividad antinflamatoria de la dexametasona más complejo B en la cirugía de terceros molares inferiores.

Métodos: El presente es un ensayo clínico controlado aleatorizado a doble ciego y de diseño en paralelo. Se llevó a cabo en la Clínica Estomatológica de la Universidad Privada Antenor Orrego (UPAO) de Trujillo durante el año 2010. La muestra estuvo conformada por 36 pacientes divididos de forma aleatoria en dos grupos. Cada paciente firmó un consentimiento informado. Una hora antes de la cirugía se administró al grupo control (A) una dosis intramuscular de dexametasona y al grupo experimental (B) una dosis intramuscular de dexametasona más complejo B. El efecto antinflamatorio se determinó por la medición horizontal de la intersección de los puntos anatómicos: trago-pogonión de tejidos blancos y gonión-ángulo externo del ojo. Las medidas se realizaron a las 24, 48 y 96 horas después de la cirugía.

Resultados: Al comparar los grupos se observó que, al administrar dexametasona más complejo B, existía una menor inflamación postoperatoria que al administrar dexametasona sola a las 24,48 y 96 horas $(p=0,03 ; p=0,002 ; p=0,001)$. Los mismos resultados se observaron para el género masculino ( $p=0,01)$, femenino $(p=0,00001)$, los menores de 25 años $(p=0,011)$ y los mayores o iguales a 25 años $(p=0,029)$.

Conclusión: La administración intramuscular de dexametasona más complejo B antes de la cirugía de terceros molares produce una menor inflamación postoperatoria y es independiente del género y la edad.
\end{abstract}

*Autor para correspondencia:

Correo electrónico: hiav_666@hotmail.com, hiav30@gmail.com (Heber Isac Arbildo Vega).

DOI: 10.20986/recom.2019.1025/2019

1130-0558/@ 2019 SECOM. Publicado por Inspira Network. Este es un artículo Open Access bajo la licencia CC BY-NC-ND (http:// creativecommons.org/licenses/by-nc-nd/4.0/). 


\section{Antiinflamatory effectiveness of dexamethasone plus B complex in lower third molar surgeries. A randomized controlled parallel double blind clinical trials}

Keywords:

Dexamethasone, third molar, oral surgery, edema, antiinflammatory effect, clinical trial.

\section{A B S T R A C T}

Objective: To determine the antiinflammatory effectiveness of dexamethasone plus complex $B$ in lower third molar surgery.

Methods: The present study is a double-blind randomized controlled trial of parallel design. It was carried out in the Stomatologic Clinic of the Universidad Privada Antenor Orrego (UPAO) of Trujillo during 2010. The sample consisted of 36 patients randomly divided into two groups. Each patient signed an informed consent. One hour prior to surgery, an intramuscular dose of dexamethasone and an intramuscular dose of dexamethasone plus complex $B$ were administered to the control group (A). The anti-inflammatory effect was determined by the horizontal measurement of the intersection of the dots anatomical: tragus-white tissue pogonion and gonion-external angle of the eye. Measurements were performed at 24, 48 and 96 hours after surgery.

Results: When comparing the groups, it was observed that the administration of dexamethasone plus B complex showed a lower postoperative inflammation than that of dexamethasone alone at 24, 48 and 96 hours $(p=0.03 ; p=0.002 ; p=0.001)$. The pivotal results were observed for males $(p=0.01)$, females $(p=0.00001)$, those under 25 years $(p=0.011)$ and greater than or equal to 25 years $(p=0.029)$.

Conclusion: Intramuscular administration of dexamethasone plus B complex prior to third molar surgery produces less post-operative inflammation and is independent of gender and age.

\section{INTRODUCCIÓN}

El tercer molar mandibular es el diente impactado más comúnmente encontrado en los seres humanos ${ }^{1-6} \mathrm{y}$ a menudo es tratado con una exodoncia quirúrgica, convirtiéndose así en una de las intervenciones más frecuentes en cirugía oral ${ }^{1,7-14}$. Se sabe que cada cirugía conlleva riesgos y que la exodoncia quirúrgica de terceros molares se relaciona a menudo con

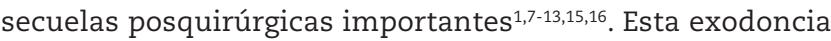
varía en dificultad y en el grado de trauma causado a los tejidos circundantes. Por ello se requiere un conocimiento confiable de la profundidad y angulación de estas piezas dentales y su relación con las estructuras vitales circundantes, así como el manejo profesional de las complicaciones quirúrgicas asociadas ${ }^{1,7-9,11,13,15,17}$.

Como cualquier procedimiento quirúrgico maxilofacial, los pacientes pueden experimentar complicaciones postoperatorias inflamatorias indeseables, algunas de las cuales pueden prevenirse o minimizarse mediante el cumplimiento de las instrucciones postoperatorias, la aplicación simultánea de crioterapia y la administración precisa de medicamentos apropiados $1,7-10,13,15,16,18,19$. Debido a esto, actualmente se utilizan medicamentos para reducir estas complicaciones, y entre ellos se incluyen los corticosteroides, que han mostrado buenos efectos inmunosupresores, antinflamatorios y analgési$\cos ^{1,7-11,13,15-23}$.

La dexametasona, un miembro de la familia de los corticosteroides y análogo de la prenidsolona, ha demostrado ser eficaz en el alivio del dolor, la hinchazón y otras complicaciones indeseables después de la cirugía de tercer molar 1,7-13,15,16,19,24. Recientemente se ha prestado atención a la administración de dexametasona intramuscular en el manejo de complicaciones quirúrgicas ${ }^{7,10,12,21,23}$ y también por vía oral ${ }^{12,13,15,16,18,23}$, submuco$\mathrm{sa}^{8,10}$ e intravenosa ${ }^{18,19}$. Sin embargo, no existe evidencia sobre el uso de dexametasona más complejo B en la reducción de estas complicaciones.

El propósito de este estudio fue determinar la efectividad antinflamatoria de la dexametasona más complejo B en la cirugía de terceros molares.

\section{MATERIALES Y MÉTODOS}

Se realizó un estudio analítico, experimental, ensayo clínico controlado aleatorizado a doble ciego y diseño en paralelo. La muestra estuvo compuesta por los pacientes que acudieron a la Clínica Estomatológica de la UPAO en Trujillo-Perú y que precisaron exodoncias de terceros molares inferiores impactados durante el año 2010. El tipo de muestreo fue probabilístico aleatorio simple.

Los criterios de inclusión para el estudio fueron: pacientes que acudieron a la Clínica Estomatológica de la UPAO en Trujillo-Perú durante el año 2010, que requerían exodoncias de terceros molares inferiores impactados que presentaban un índice de dificultad moderado según el índice de dificultad de Pederson ${ }^{25}$, pacientes con un aparente buen estado de salud general y que aceptaran el tratamiento farmacológico bajo consentimiento informado. Los criterios de exclusión fueron: pacientes con riesgo sistémico no controlado, gestantes o en periodo de lactancia, pacientes con enfermedades infectocontagiosas, con alguna incapacidad física o mental, con alguna patología dentoalveolar o periodontal, menores de 18 años, con alergia a los fármacos que fueron aplicados, que hubieran tomado algún analgésico durante las últimas 
24 horas, que no hubieran aceptado participar en el estudio y que lo manifestasen y pacientes con ansiedad.

Para la realización del estudio se tuvo en cuenta la declaración de Helsinki (2008) de la Asamblea Médica Mundial, de un jurado perteneciente a la Escuela de Posgrado del Área de Ciencias Médicas de la Universidad Nacional de Trujillo, de la Ley General de Salud en el Perú (Ley n. ${ }^{\circ}$ 26842) y de la autorización de cada participante en el estudio (consentimiento informado). Se calculó el tamaño de la muestra y se obtuvo que era necesario seleccionar 36 pacientes.

Se efectuaron, en primer lugar, los trámites documentarios correspondientes para conseguir la autorización del director de la Clínica Estomatológica de la UPAO para la realización del estudio en dicho lugar. Posteriormente se comenzó a seleccionar a los pacientes según los criterios de inclusión y exclusión mencionados, para luego distribuirlos en dos grupos (grupo A o control y grupo B o experimental) de forma aleatoria por medio de un generador de números aleatorios por ordenador. A los pacientes del grupo A (18 participantes, 10 hombres y 8 mujeres) se les administró, una hora antes de la cirugía, una ampolla de dexametasona de $4 \mathrm{mg}$ por vía intramuscular, y a los pacientes del grupo B (18 participantes, 12 hombres y 6 mujeres) se les administró, una hora antes de la cirugía, una ampolla de Dexaneurobion ${ }^{\circledR}$ (dexametasona + vitamina $B_{1}$ + vitamina $B_{6}$ + vitamina $B_{12}$ ) por vía intramuscular, con el objetivo de valorar la efectividad antinflamatoria postoperatoria. Cada fármaco fue administrado por una enfermera con 5 años de experiencia en el ámbito clínico y era la única que sabía, en primera instancia, qué fármaco recibía cada paciente. Posteriormente, al final del análisis estadístico se le preguntó qué fármaco había recibido cada participante. Los fármacos que se utilizaron son productos comercializados y de amplio uso en odontología.

Cada participante tuvo conocimiento de qué se trataba el estudio, para lo cual cada uno firmó un consentimiento informado y leyó la hoja explicativa del estudio antes de empezar. Una vez realizado esto, el operador, que fue un especialista en Cirugía Oral y Maxilofacial, comenzó a realizar la exodoncia de cada tercer molar impactado con materiales completamente estériles. Tanto los participantes como el examinador, el operador y posteriormente el estadístico no conocían el orden ni la marca comercial de los fármacos que se administraron a cada paciente.

Una vez terminada la cirugía se despidió al participante y se le citó a las 24, 48 y 96 horas para realizar controles. En cada control el examinador tuvo una ficha de recolección de datos que constaba de dos apartados: datos del participante (género, edad y fecha) y un cuadro en donde se anotaron los datos correspondientes a la medición del edema (medidas a las 24, 48 y 96 horas después de la cirugía), que fueron expresadas en centímetros.

Para la medición del edema, el examinador contaba con: un calibrador para la medición de la parte externa de la cara, un calibrador Vernier y un plumón indeleble; y se realizó de la siguiente manera: en ambas mejillas se procedió a marcar los puntos trago (T), gonión (Go), pogonión (Pg) y ángulo externo del ojo (A); luego, se procedió a trazar los planos T-Pg y Go-A uniendo los puntos que pertenecen a cada plano y así marcar la intersección de ambos planos; posteriormente, con el calibrador para la medición de la parte externa de la cara se midió, con ayuda del calibrador Vernier, el ancho existente entre los puntos de intersección del lado derecho e izquierdo de la cara, y finalmente se anotó dicha medición en la ficha de recolección de datos.

Los datos recolectados fueron introducidos en una base de datos y posteriormente procesados empleando el programa SPSS 17.0. Se refirieron las medias para cada fármaco administrado por participantes con sus correspondientes desviaciones estándar. La comparación de la efectividad de los fármacos fue analizada mediante la prueba t de Student para dos muestras independientes. La significación estadística fue del $5 \%$.

\section{RESULTADOS}

De los 120 pacientes examinados en un inicio, se eligieron 36 pacientes que cumplían con los criterios de inclusión y exclusión y fueron analizados hasta el final del estudio (Figura 1).

La distribución de la muestra según su género y edad se puede observar en la Tabla I. Al comparar la efectividad antinflamatoria de cada uno de los fármacos se encontró que existe una diferencia estadísticamente significativa a las 24,48 y 96 horas después de la cirugía ( $p=0,028, p=0,002$ y $p=0,001$ ) (Tabla II). Al comparar la efectividad antinflamatoria de cada uno de los fármacos en los hombres, se encontró que existe una diferencia estadísticamente significativa a las 24, 48 y 96 horas después de la cirugía ( $p=0,026, p=0,003$ y $p=0,01$ ) (Tabla III). Al comparar la efectividad antinflamatoria de cada uno de los fármacos en las mujeres, se encontró que no existe una diferencia estadísticamente significativa a las 24 horas después de la cirugía $(p=0,056)$, pero sí existe una diferencia estadísticamente significativa a las 48 y 96 horas después de la cirugía ( $p=0,02$ y $p=0,00001$ ) (Tabla III). Al comparar la efectividad antinflamatoria de cada uno de los fármacos en los participantes menores de 25 años, se encontró que no existe una diferencia estadísticamente significativa a las 24 horas después de la cirugía $(p=0,102)$, pero sí existe una diferencia estadísticamente significativa a las 48 y 96 horas después de la cirugía ( $p=0,016$ y $p=0,011$ ) (Tabla III). Al comparar la efectividad antinflamatoria de cada uno de los fármacos en los participantes mayores de 25 años, se encontró que no existe una diferencia estadísticamente significativa a las 24 horas después de la cirugía $(p=0,305)$, pero sí existe una diferencia estadísticamente significativa a las 48 y 96 horas después de la cirugía ( $p=0,041$ y $p=0,029)$ (Tabla III).

\section{DISCUSIÓN}

En el presente ensayo clínico aleatorizado se detectó una mayor reducción de la inflamación con la dexametasona y el complejo B, que fue estadísticamente significativa. Hasta donde sabemos no se han publicado informes que reporten la efectividad antinflamatoria preoperatoria de la dexametasona con el complejo B en el tratamiento quirúrgico de los terceros molares inferiores para reducir la inflamación y su comparación con la dexametasona. Por lo tanto, una comparación directa con otros estudios no fue posible.

Si bien es cierto que la bibliografía actual menciona que el uso del complejo B es un excelente ayudante en la reducción del dolor y que dentro del proceso inflamatorio también existe 


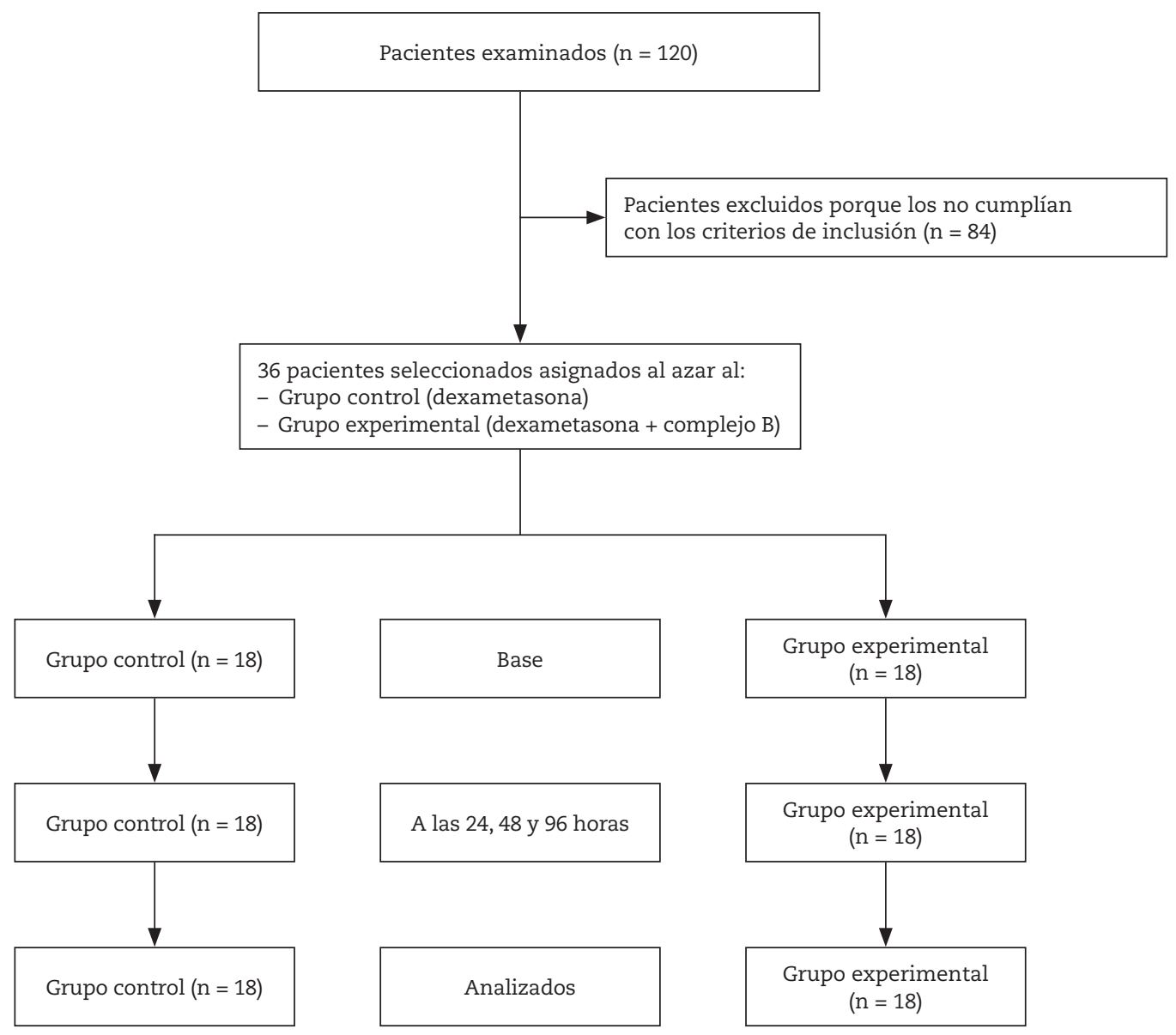

Figura 1. Diagrama de flujo del estudio (según CONSORT).

\begin{tabular}{lccc}
\multirow{2}{*}{ Tabla I. } & \multicolumn{2}{c}{ Distribución de pacientes según género y edad } \\
\hline & Variable & $\mathrm{n}$ & $\%$ \\
\hline \multirow{2}{*}{ Género } & Hombre & 22 & 61,1 \\
& Mujer & 14 & 38,9 \\
\multirow{2}{*}{ Edad } & $<25$ años & 30 & 83,3 \\
& $\geq 25$ años & 6 & 16,7 \\
\hline
\end{tabular}

el dolor, puede darse la posibilidad de que este mismo compuesto ayude no solamente en la analgesia sino también en la reducción de la inflamación; sin embargo, para llegar a esta afirmación se tendrían que realizar más estudios que logren corroborar esta hipótesis.

Las características de la muestra estudiada, en cuanto al género, presentan heterogeneidad, ya que existe un mayor número de hombres que de mujeres. El predominio de este grupo posiblemente se deba a que en el periodo de realización del estudio acudieron a la Clínica Estomatológica de la UPAO en Trujillo-Perú más hombres que mujeres, o que al analizar el índice de dificultad de Pederson quienes más cumplían el criterio de inclusión "moderado" eran los hombres.

En relación con la edad, la muestra presenta una heterogeneidad muy marcada, ya que existe un mayor predominio de pacientes menores de 25 años. El predominio de este grupo probablemente se deba a que las personas jóvenes presentaron mayores molestias a causa de los terceros molares; sin embargo, existe un pequeño grupo de personas que no presentan dichas molestias, pero que precisan de la exodoncia de los terceros molares para prevenir futuras complicaciones en las piezas dentales subsiguientes.

En cuanto al género y la edad, se observa que tanto en hombres como en mujeres y tanto en jóvenes como en adultos existe una mayor reducción de la inflamación al administrar de forma preoperatoria la dexametasona con el complejo B que cuando se administra de forma preoperatoria solamente la dexametasona. Esto nos da a entender que el uso de este fármaco es efectivo de forma independiente del género y la edad, lo que posiblemente se deba al efecto añadido que puede proporcionar el complejo B a la dexametasona.

Una de las desventajas que podemos encontrar en el presente estudio es la realización de una comparación biológica (edema) en personas distintas (ensayo clínico en paralelo), ya que, como se sabe, cada persona tiene una reacción distinta ante las lesiones y posteriormente ante el edema y el dolor. Sin embargo, el estudio cuenta con una notable ventaja, y es que los terceros molares inferiores de cada uno de pacientes presentaban un índice de dificultad interme- 
Tabla II. Efectividad antinflamatoria de la dexametasona y la dexametasona más complejo B en la cirugía de terceros molares inferiores

\begin{tabular}{llllll}
$\begin{array}{c}\text { Tiempo de evaluación } \\
\text { postoperatoria }\end{array}$ & \multicolumn{1}{c}{ Grupos } & $\mathrm{n}$ & Media & Desviación estándar & $p^{*}$ \\
\hline \multirow{2}{*}{24 horas } & Control & 18 & 12,951 & 0.605 & 0,028 \\
& Experimental & 18 & 12,495 & 0,585 & 0,537 \\
\multirow{2}{*}{48 horas } & Control & 18 & 12,987 & 0,549 & 0,002 \\
& Experimental & 18 & 12,369 & 0,485 & 0,093 \\
96 horas & Control & 18 & 12,675 & 11,979 & 0,001 \\
\hline
\end{tabular}

${ }^{*}$ Prueba $t$ de Student para dos muestras independientes.

Tabla III. Efectividad antinflamatoria de la dexametasona y la dexametasona más complejo B en la cirugía de terceros molares inferiores según género y edad

\begin{tabular}{|c|c|c|c|c|c|c|}
\hline Género & $\begin{array}{c}\text { Tiempo } \\
\text { de evaluación } \\
\text { postoperatoria }\end{array}$ & Grupos & $\mathrm{n}$ & Media & $\begin{array}{c}\text { Desviación } \\
\text { estándar }\end{array}$ & $p^{*}$ \\
\hline \multirow{6}{*}{ Hombres } & \multirow{2}{*}{24 horas } & Control & 10 & 13.282 & 0,527 & \multirow{2}{*}{0,026} \\
\hline & & Experimental & 12 & 12,677 & 0,631 & \\
\hline & \multirow{2}{*}{48 horas } & Control & 10 & 13,29 & 0,441 & \multirow{2}{*}{0,003} \\
\hline & & Experimental & 12 & 12,513 & 0,593 & \\
\hline & \multirow{2}{*}{96 horas } & Control & 10 & 12,932 & 0,443 & \multirow{2}{*}{0,01} \\
\hline & & Experimental & 12 & 12,265 & 0,626 & \\
\hline \multirow{6}{*}{ Mujeres } & \multirow{2}{*}{24 horas } & Control & 8 & 12,536 & 0,422 & \multirow{2}{*}{0,056} \\
\hline & & Experimental & 6 & 12,132 & 0,225 & \\
\hline & \multirow{2}{*}{48 horas } & Control & 8 & 12,608 & 0,394 & \multirow{2}{*}{0,02} \\
\hline & & Experimental & 6 & 12,082 & 0,317 & \\
\hline & \multirow{2}{*}{96 horas } & Control & 8 & 12,354 & 0,327 & \multirow{2}{*}{0,00001} \\
\hline & & Experimental & 6 & 11,407 & 0,426 & \\
\hline \multirow{6}{*}{$<25$ años } & \multirow{2}{*}{24 horas } & Control & 14 & 12,826 & 0,462 & \multirow[t]{2}{*}{0,102} \\
\hline & & Experimental & 16 & 12,484 & 0,62 & \\
\hline & \multirow{2}{*}{48 horas } & Control & 14 & 12,86 & 0,463 & \multirow[t]{2}{*}{0,016} \\
\hline & & Experimental & 16 & 12,364 & 0,583 & \\
\hline & \multirow{2}{*}{96 horas } & Control & 14 & 12,562 & 0,114 & \multirow[t]{2}{*}{0,011} \\
\hline & & Experimental & 16 & 11,973 & 0,184 & \\
\hline \multirow{6}{*}{$\geq 25$ años } & \multirow{2}{*}{24 horas } & Control & 8 & 13,388 & 0,906 & \multirow[t]{2}{*}{0,305} \\
\hline & & Experimental & 6 & 12,585 & 0,163 & \\
\hline & \multirow{2}{*}{48 horas } & Control & 8 & 13,43 & 0,607 & \multirow[t]{2}{*}{0,041} \\
\hline & & Experimental & 6 & 12,415 & 0,12 & \\
\hline & \multirow{2}{*}{96 horas } & Control & 8 & 13,07 & 0,53 & \multirow[t]{2}{*}{0,029} \\
\hline & & Experimental & 6 & 12,03 & 0,028 & \\
\hline
\end{tabular}

${ }^{*}$ Prueba $t$ de Student para dos muestras independientes.

dia, por lo que la lesión en la zona quirúrgica y el tiempo operatorio fueron mayores, lo que conllevaría una notable respuesta inflamatoria de toda la zona quirúrgica, de forma que la mayor disminución de este proceso inflamatorio mediante la administración preoperatoria de un fármaco que reduzca este efecto es un gran avance para la odontología.

Adicionalmente, los autores creemos que este estudio es un inicio para la realización de futuras investigaciones que comparen la acción farmacológica de estos dos fármacos en una misma persona (ensayo clínico cruzado) y de la comparación de la dexametasona más complejo B con otros fármacos antinflamatorios.

\section{CONCLUSIÓN}

La administración intramuscular de dexametasona más complejo B antes de la cirugía de terceros molares produce una menor inflamación postoperatoria que la administración 
intramuscular de dexametasona sola, y esto es independiente del género y la edad.

\section{FINANCIACIÓN}

Autofinanciado.

\section{CONFLICTOS DE INTERÉS}

Los autores declaran no tener conflicto de interés en relación con los resultados publicados.

\section{B I B L I O G R A F Í A}

1. Al-Dajani M. Can preoperative intramuscular single-dose dexamethasone improve patient-centered outcomes following third molar surgery? J Oral Maxillofac Surg. 2017;75(8):1616-26. DOI: 10.1016/j.joms.2017.03.037.

2. Pedro FL, Bandeca MC, Volpato LE, Marques AT, Borba AM, Musis CR, et al. Prevalence of impacted teeth in a Brazilian subpopulation. J Contemp Dent Pract. 2014;15(2):209-13.

3. Cassetta M, Altieri F, Di Mambro A, Galluccio G, Barbato E. Impaction of permanent mandibular second molar: a retrospective study. Med Oral Patol Oral Cir Bucal. 2013;18(4):e564-8. DOI: 10.4317/medoral.18869.

4. Padhye MN, Dabir AV, Girotra CS, Pandhi VH. Pattern of mandibular third molar impaction in the Indian population: a retrospective clinico-radiographic survey. Oral Surg Oral Med Oral Pathol Oral Radiol. 2013;116(3):e161-6. DOI: 10.1016/j.oooo.2011.12.019.

5. Hashemipour MA, Tahmasbi-Arashlow M, Fahimi-Hanzaei F. Incidence of impacted mandibular and maxillary third molars: a radiographic study in a Southeast Iran population. Med Oral Patol Oral Cir Bucal. 2013;18(1):e140-5. DOI: 10.4317/medoral.18028.

6. Yilmaz S, Adisen MZ, Misirlioglu M, Yorubulut S. Assessment of Third molar impaction pattern and associated clinical symptoms in Central Anatolian Turkish population. Med Princ Pract. 2016;25(2):169-75. DOI: 10.1159/000442416.

7. Rocha-Neto AM, Nogueira EF, Borba PM, Laureano-Filho JR, Vasconcelos BC. Application of dexamethasone in the masseter muscle during the surgical removal of lower third molars. J Craniofac Surg. 2017;28(1):e43-7. DOI: 10.1097/SCS.0000000000003188.

8. Nair RB, Rahman NM, Ummar M, Hafiz KA, Issac JK, Sameer KM. Effect of submucosal injection of dexamethasone on postoperative discomfort after third molar surgery: a prospective study. J Comtemp Dent Pract. 2013;14(3):401-4.

9. Mehra P, Reebye U, Nadershah M, Cottrell D. Efficacy of antiinflammatory drugs in third molar surgery: a randomized clinical trial. Int J Oral Maxillofac Surg. 2013;42(7):835-42. DOI: 10.1016/j.ijom.2013.02.017.

10. Mojsa IM, Pokrowiecki R, Lipczynski K, Czerwonka D, Szczeklik $\mathrm{K}$, Zaleska M. Effect of submucosal dexamethasone injection on postoperative pain, oedema, and trismus following mandibular third molar surgery: a prospective, randomized, double-blind clinical trial. Int J Oral Maxillofac Surg. 2017;46(4):52430. DOI: 10.1016/j.ijom.2016.11.006.

11. Sabhlok S, Kenjale P, Mony D, Khatri I, Kumar P. Randomized controlled trial to evaluate the efficacy of oral dexamethasone and intramuscular dexamethasone in mandibular third molar surgeries. J Clin Diagn Res. 2015;9(11):ZC48-51. DOI: 10.7860/ JCDR/2015/13930.6813.
12. Murugesan K, Sreekumar K, Sabapathy B. Comparison of the roles of serratiopeptidase and dexamethasone in the control of inflammation and trismus following impacted third molar surgery. Indian J Dent Res. 2012;23(6):709-13. DOI: 10.4103/09709290.111243

13. Al-Shamiri HM, Shawky M, Hassanein N. Comparative assessment of preoperative versus postoperative dexamethasone on postoperative complications following lower third molar surgical extraction. Int J Dent. 2017;2017:1350375. DOI: 10.1155/2017/1350375.

14. Aravena PC, Cartes-Velásquez R, Rosas C. Signs and symptoms of postoperative complications in third molar surgery. J Int Dent Med Res. 2015;8(3):140-6.

15. Lima CAA, Favarini VT, Torres AM, da Silva RA, Sato FRL. Oral dexamethasone decreases postoperative pain, swelling, and trismus more than diclofenac following third molar removal: a randomized controlled clinical trial. Oral Maxillofac Surg. 2017;21(3):321-6. DOI: 10.1007/s10006-017-0635-0.

16. Bortoluzzi MC, Capella DL, Barbieri T, Pagliarini M, Cavalieri T, Manfro R. A single dose of amoxicillin and dexamethasone for prevention of postoperative complications in third molar surgery: a randomized, double-blind, placebo controlled clinical trial. J Clin Med Res. 2013;5(1):26-33. DOI: 10.4021/jocmr1160w.

17. Kim K, Brar P, Jakubowski J, Kaltman S, Lopez E. The use of corticosteroids and nonsteroidal antiinflammatory medication for the management of pain and inflammation after third molar surgery: a review of the literature. Oral Surg Oral Med Oral Pathol Oral Radiol Endod. 2009;107(5):630-40. DOI: 10.1016/j.tripleo.2008.11.005.

18. Chaudhary PD, Rastogi S, Gupta P, Niranjanaprasad Indra B, Thomas R, Choudhury R. Pre-emptive effect of dexamethasone injection and consumption on post-operative swelling, pain, and trismus after third molar surgery. A prospective, double blind and randomized study. J Oral Biol Craniofac Res. 2015;5(1):21-7. DOI: 10.1016/j.jobcr.2015.02.001.

19. Arakeri G, Rai KK, Shivakumar HR, Jayade B. A randomized clinical trial to compare the efficacy of submucosal aprotinin injection and intravenous dexamethasone in reducing pain and swelling after third molar surgery: a prospective study. J Maxillofac Oral Surg. 2013;12(1):73-9. DOI: 10.1007/s12663-0120364-8.

20. Hargreaves KM, Costello A. Glucocorticoids suppress levels of immunoreactive bradykinin in inflamed tissue as evaluated by microdialysis probes. Clin Pharmacol Ther. 1990;48(2):16878. DOI: 10.1038/clpt.1990.132.

21. Ferrari AM, Byers MR. Chronic dexamethasone treatment and its effects on sensory neuropeptides, pulpal injury reactions and reparative dentin. Brain Res. 1996;723(1-2):125-34. DOI: 10.1016/0006-8993(96)00231-4.

22. Klongnoi B, Kaewpradub P, Boonsiriseth K, Wongsirichat N. Effect of single dose preoperative intramuscular dexamethasone injection on lower impacted third molar surgery. Int J Oral Maxillofac Surg. 2012;41(3):376-9. DOI: 10.1016/j. ijom.2011.12.014.

23. Simone JL, Jorge WA, Horliana AC, Canaval TG, Tortamano IP. Comparative analysis of preemptive analgesic effect of dexamethasone and diclofenac following third molar surgery. Braz Oral Res. 2013;27(3):266-71. DOI: 10.1590/S1806-83242013005000012.

24. Boonsiriseth $\mathrm{K}$, Klongnoi B, Sirintawat N, Saengsirinavin C, Wongsirichat N. Comparative study of the effect of dexamethasone injection and consumption in lower third molar surgery. Int J Oral Maxillofac Surg. 2012;41(2):244-7. DOI: 10.1016/j. ijom.2011.12.011.

25. Yuasa H, Kawai T, Sugiura M. Classification of surgical difficulty in extracting impacted third molars. Br J Oral Maxillofac Surg. 2002;40(1):26-31. DOI: 10.1054/bjom.2001.0684. 